\title{
Day-ahead Hourly Electricity Load Modeling by Functional Regression
}

\author{
Yonghan Feng ${ }^{1, *}$, Sarah M. Ryan ${ }^{2}$
}

\begin{abstract}
Short-term load forecasting is important for power system generation planning and operation. For unit commitment and dispatch processes to incorporate uncertainty, a short-term load model must not only provide accurate load predictions but also enable the generation of reasonable probabilistic scenarios or uncertainty sets. This paper proposes a temporal and weather conditional epi-splines based load model (TWE) using functional approximation. TWE models the dependence of load on time and weather separately by functional approximation using epi-splines, conditional on season and area, in each segment of similar weather days. Load data are transformed from various day types to a specified reference day type among similar weather days in the same season and area, in order to enrich the data for capturing the non-weather dependent load pattern. In an instance derived from an Independent System Operator in the U.S., TWE not only provides accurate hourly load prediction and narrow bands of prediction errors, but also yields serial correlations among forecast hourly load values within a day that are similar to those of actual hourly load.
\end{abstract}

Keywords: Short-term load model, Forecasting, Day-ahead scenario, Epi-splines

\footnotetext{
*Corresponding author

Email addresses: yhfeng.isu@gmail.com (Yonghan Feng ), smryan@iastate.edu (Sarah M. Ryan )

${ }^{1}$ Sears Holdings Corporation, Hoffman Estates, IL 60179, USA

${ }^{2}$ Industrial and Manufacturing System Engineering Department, Iowa State University, Ames, IA 50011, USA
} 


\section{Introduction}

Electricity load forecasting significantly influences planning and operation of power systems. Different models are used for long-term [1], medium-term [2], and short-term forecasting [3, 4]. Short-term load forecasting (STLF) applies to horizons from an hour to a day ahead, and forms the basis for unit commitment, economic dispatch, maintenance plans for generators, and electricity price forecasting for utilities and independent system operators $[4,5,6]$. Because of its essential role in power systems operations, inaccurate load forecasting can cause high operational and generation cost, equipment failure, or even system blackout. As emphasis on efficient and robust scheduling of thermal generators with uncertain load prediction increases, stochastic programming and robust optimization have been extensively studied for use in the operation of power systems $[7,8,9,10,11,12,13,14]$. In the context of stochastic/robust unit commitment or economic dispatch by utilities or independent system operators, a regional short-term load model must provide not only accurate hour-by-hour point forecasts but also intervals and correlations to generate reasonable probabilistic scenario trajectories or ranges from which to form uncertainty sets.

A broad literature on STLF has developed for power systems of various scales. This paper focuses on regional short-term load forecasting. At the regional level, in addition to the nonstationarity in mean and variance as well as seasonal patterns, a number of external factors such as weather, time, economic activity, and social habits complicate the SLTF problem with nonlinearity. Various modeling approaches have been proposed to address these difficulties. Most of the short-term load modeling approaches can be categorized as either classical statistical methods or machine learning (ML) methods. The former often presume that load is a function of several explanatory variables; e.g., previous load values, temperature and humidity, and then estimate parameters of the specified function. In contrast, ML methods do not restrict themselves to specified functions. The ML methods include artificial neural networks $[15,16]$ and support vector machines $[17,18,19]$. See $[4,20,21]$ for broad reviews of ML methods. Hybrids of statistical and ML methods have also been applied recently [22, 23, 24, 25]. Similar day methods are often used by system operators and utilities to forecast future load and wind energy production $[26,27]$. In these methods, historical days with weather and day type similar to the target day are identified, and the actual load of a similar day is taken as a forecast. Such methods are often 
embedded in a more complicated load model, as in [16], because the methods do not sufficiently capture complex load features if used alone. Regarding smaller scale power systems including residential and commercial areas, as well as the building level, hybrids of multiple regression, time series, and ML based methods are often used [28, 29, 30].

Among statistical methods, time series and regression methods are widely used to build short-term load models. The autoregressive moving average (ARMA) model is one of the most frequently used time series methods [31, 32, $33,34,35,36]$. In addition to a Box-Jenkins time series model, Uri improved the short-term load prediction accuracy by taking into account price and weather change [31]. Amjady identified different ARMA load models for hot days and cold days while considering effects from weekdays and weekends on daily load patterns, as well as the temperature effect on load in [32]. Huang and Shih presented a modified univariate ARMA short-term load model by considering a non-Gaussian process in [33]. Taylor first established a univariate time series load model which considered within-day and withinweek seasonality by using exponential smoothing in [34]. He then improved the load prediction by accounting for the intraday, intraweek, and intrayear effects of short-term load in Britain and France without external factors [35]. Liu et al. improved upon the previous work by estimating the effects of temperature, hour-of-day and type-of-day on load level using nonparametric regression, and employed an univariate ARMA model to model the load residuals caused by the nonparametric regression [36]. In general, time series methods predict well for the immediate future, but they may suffer from imprecision in the multi-step ahead predictions because of the accumulated prediction errors.

Regression on various explanatory variables is another major direction of short-term load modeling. Usually, regression models for load forecasting include weather conditions, day types, holidays, economic conditions, and social habits. The early literature considered only impacts of temperature and holidays on load [37, 38]. Haida and Muto applied transformations to include load changes in recent days and seasons, as well as annual load growth in a linear regression model [39]. Charytoniuk et al. considered load forecast as a local average of observed past loads within the local neighborhood and the specific weights on the loads defined by a multivariate product kernel [40]. Aldo et al. in [5] classified daily load curves by functional clustering, and then developed a family of functional linear regression models on the basis of obtained groups. Hong presented a multiple linear regression model 
that considered load as a polynomial function of temperature for each hour in each day type in each month [4]. Black also applied multiple linear regression to examine the influence of weather on load in [41], but focused on summer weekdays in the region served by the Independent System Operator of New England (ISO-NE). In the most recent published results of competitive energy forecasting, Charlton and Singleton in [42] refined the multiple linear regression model of [4], and achieved small prediction errors and weighted root mean square error (WRMSE) simultaneously by taking into account multiple weather stations, day-of-season effects and smoother temperature forecasts. Fan and Hyndman proposed a semi-parametric additive model [43], which applied cubic splines to estimate the relationship between load and temperature as well as previous loads. Most of the multiple regression methods mentioned here achieved encouraging point predictions by establishing a load model for each hour (or each half hour) in a day but, with the exception of [43], did not take into account or assess the distributions of hourly forecasts.

Although a number of load backcasting studies have presented encouraging matches to actual loads using actual weather records as input, the associated load models may not be satisfactory for predicting a day-long trajectory of hourly load or for constructing probabilistic load scenarios or uncertainty sets for day-ahead unit commitment. Even if the model accurately captures the effects of actual weather on electricity demand, the imperfect day-ahead weather forecast could distort the corresponding load forecast. To ensure that the load forecast error distribution would reflect the imprecision in both weather forecast and the modeled relationship between weather and load, models were constructed based on the day-ahead weather forecast that is available to utilities and independent system operators [44, 45]. These models formed the basis for probabilistic scenarios as daylong trajectories of hourly loads [46]. Similarly to [44, 45], we propose a regional day-ahead 24-hour load prediction model based on the next day hourly weather forecast, but we assess the hourly non-weather dependent load component by means of an error-minimizing procedure instead of using average hourly load as in [46]. Due to its improved accuracy, the resulting load forecast is more appropriate for creating practical load scenarios.

The main contribution of this paper is a short-term load model that starts from a weather forecast and uses functional approximation rather than ordinary regression to model the complex nonlinearity between weather (i.e., temperature and dew point temperature) forecast and electricity consump- 
tion levels in multiple geographic zones. By capturing the temporal load patterns in segments of enriched data, it not only accurately predicts hourly load values but produces serial correlations among them that are similar to those of actual hourly load trajectories. The model is supported by segmenting the input data according to seasonal calendar effects on electricity consumption and by enriching the data in each segment to enhance the prediction capability. Calendar months are grouped into seasons according to monthly load-temperature patterns using $k$-means clustering. Hourly load data for a specified day type are enriched using linear transformation among different day types. In a case study derived from ISO-NE, when applied to historical weather forecast data the proposed short-term load model leads to a narrower and less skewed distribution of prediction errors for each zone in each season, compared to two recent regression based short-term load models $[4,42]$.

The rest of the paper is organized as follows. After an overview of the data processing and modeling steps, Section 2 develops a weather conditional short-term load model by applying an epi-spline approximation technique. To demonstrate its regional application, Section 3 describes the partitioning of data into several segments according to calendar effects (day types and seasons), and enrichment by transformation to a reference day type. The numerical results in Section 4 indicate that our model captures the complex nonlinear relationship between electricity consumption level and weather conditions, and attains narrower and less skewed distributions of prediction errors, compared to those of two benchmark models. Section 5 concludes this paper.

\section{Day-ahead hourly load model}

In this paper, day-ahead hourly load models are developed for multiple zones within a region based on their corresponding weather forecasts. The temporal relationship between weather forecast and electricity consumption changes during the year because of its use for heating in winter and cooling in summer, as well as diurnal lighting patterns. As discussed in $[4,32,36]$, the accuracy of load prediction could be improved if calendar month or season effects are considered in a load model. In this paper, calendar months with similar load-temperature patterns are grouped together to form an artificial season. In the same artificial season, days are further divided into three weather segments according to daily average temperature to enforce the sim- 
ilarity in the same segment. Due to the limited load data records in each segment, and the similarity among daily load sequences in the same weather segment in the same season, load data are enriched by transforming various day types to the specified reference day type, to enhance the prediction capability. In addition to temperature, humidity and temporal dependence are other important factors to predict the 24-hour sequence of load values in the next day. The data analysis and transformation steps are detailed in the context of a case study in Section 3.

Motivated by the power of epi-splines to derive term and volatility structures associated with financial markets [47], and their successful applications in numerous areas [48], in this paper epi-splines are applied separately in each segment of a season for each zone to model the complex nonlinear temporal dependence of load and effects of weather on load. Section 2.1 introduces epi-splines. Section 2.2 proposes a short-term load model based on weather forecast and temporal dependence.

\subsection{Approximation by epi-spline}

An epi-spline approximates a twice-continuous differentiable function $f(r)$ over an interval $[0, \varrho]$ (note that any interval $[\alpha, \beta]$ can be transformed to such an interval). The whole interval $[0, \varrho]$ is partitioned into $K$ equal subintervals $\mathcal{A}_{k}=\left(r_{k-1}, r_{k}\right]$ where $r_{0}=0$ and $r_{k}=r_{k-1}+\delta, k=1, \cdots, K$. By applying a piece-wise constant function

$$
a(r)=a_{k}, r \in \mathcal{A}_{k}
$$

to approximate the second derivative $f^{\prime \prime}(r)$ over each subinterval $\mathcal{A}_{k}$, the first derivative $f^{\prime}(r), r \in \mathcal{A}_{k}$, is approximated by

$$
s^{\prime}(r)=w_{0}+\int_{0}^{r} a(\eta) d \eta=w_{0}+\delta \sum_{i=1}^{k-1} a_{i}+\left(r-r_{k-1}\right) a_{k} .
$$

Furthermore, the function $f(r), r \in \mathcal{A}_{k}$, is approximated by integrating 
$s^{\prime}(r)$ :

$$
\begin{aligned}
s(r) & =v_{0}+\int_{0}^{r} s^{\prime}(\eta) d \eta=v_{0}+\sum_{i=1}^{k-1} \int_{r_{i-1}}^{r_{i}} s^{\prime}(\eta) d \eta+\int_{r_{k-1}}^{r} s^{\prime}(\eta) d \eta \\
& =v_{0}+w_{0} r+\delta \sum_{i=1}^{k-1}\left(r-r_{i-1}+\frac{\delta}{2}\right) a_{i}+\frac{1}{2}\left(r-r_{k-1}\right)^{2} a_{k}
\end{aligned}
$$

where $v_{0}$ and $w_{0}$ are constants. In particular, if $r=r_{k},(3)$ can be simplified as:

$$
s\left(r_{k}\right)=v_{0}+r_{k} w_{0}+\delta^{2} \sum_{i=1}^{k}\left(k-i+\frac{1}{2}\right) a_{i} .
$$

Supposing that the influence of a weather variable, such as temperature, on load can be described as a continuous differentiable function of time, an epi-spline can be used to model this effect by following (4). The following sections present a short-term load model that considers weather influences and temporal effects on electricity demand.

\subsection{Epi-splines based load model}

Throughout this paper, let $\mathcal{M}$ denote the set of day segments in a given season, $\mathcal{J}_{m}$ the set of days of a given segment $m \in \mathcal{M}$ in a given season in training set, and $\mathcal{H}=\{1,2, \cdots, 24\}$ the set of hours in a day. Let each hour in a day $j \in \mathcal{J}_{m}$ be divided into $\gamma$ equal subintervals, with $\gamma=1 / \delta$. Let $l_{j, m}^{h}$ be the actual load in hour $h \in \mathcal{H}$ in day $j \in \mathcal{J}_{m}$ in segment $m \in \mathcal{M}$, and $t_{j, m}^{h}$ and $d_{j, m}^{h}$ be the corresponding temperature and dew point temperature forecasts from the day ahead, respectively.

\subsubsection{Temporal and weather conditional epi-splines (TWE) based load model}

The impacts from temperature and dew point temperature on electricity consumption for $h \in \mathcal{H}$ are approximated by epi-splines $s_{t, m}(h)$ and $s_{d, m}(h)$, respectively, by following (4). Because these weather variables may have different magnitudes of impact on electricity consumption in a specified season, the pre-specified constants $\mu_{t}$ and $\mu_{d}$ are applied to weight such impacts. The model proposed in this section estimates the non-weather dependent load component by including an additional epi-spline in an error-minimization model. Because the non-weather dependent load component usually changes 
with time, the additional epi-spline, denoted by $s_{m}(h)$, is a function of time only for a specified segment $m \in \mathcal{M}$. The TWE model is given by:

$$
l_{j, m}^{h}=s_{m}(h)+\mu_{t} s_{m, t}(h) t_{j, m}^{h}+\mu_{d} s_{d, m}(h) d_{j, m}^{h}+e_{j, m}^{h}, \forall h \in \mathcal{H}, \forall j \in \mathcal{J}_{m}
$$

where $e_{j, m}^{h}$ represents the fitted model error in hour $h$ of day $j$ in segment $m$. The related parameters of the load model are identified by minimizing $\left\|e_{j, m}^{h}\right\|_{1}$, in a linear program as follows:

$$
\begin{gathered}
\min _{\boldsymbol{v}_{\mathbf{0}}, \boldsymbol{w}_{\mathbf{0}}, \boldsymbol{a}, \boldsymbol{b}, \boldsymbol{c}} \sum_{j \in \mathcal{J}_{m}} \sum_{h=1}^{24} \epsilon_{j, m}^{h} \\
\text { s.t. } \epsilon_{j, m}^{h} \geq l_{j, m}^{h}-\left(v_{0, m}^{h}+h w_{0, m}^{h}+\frac{1}{\gamma^{2}} \sum_{i=1}^{\gamma h}\left(\gamma h-i+\frac{1}{2}\right) a_{i, m}\right) \\
-\mu_{t}\left(v_{0, m}^{t}+h w_{0, m}^{t}+\frac{1}{\gamma^{2}} \sum_{i=1}^{\gamma h}\left(\gamma h-i+\frac{1}{2}\right) b_{i, m}\right) t_{j, m}^{h} \\
-\mu_{d}\left(v_{0, m}^{d}+h w_{0, m}^{d}+\frac{1}{\gamma^{2}} \sum_{i=1}^{\gamma h}\left(\gamma h-i+\frac{1}{2}\right) c_{i, m}\right) d_{j, m}^{h}, \\
\forall j \in \mathcal{J}_{m}, h \in \mathcal{H} \\
\epsilon_{j, m}^{h} \geq-l_{j, m}^{h}+\left(v_{0, m}^{h}+h w_{0, m}^{h}+\frac{1}{\gamma^{2}} \sum_{i=1}^{\gamma h}\left(\gamma h-i+\frac{1}{2}\right) a_{i, m}\right) \\
+\mu_{t}\left(v_{0, m}^{t}+h w_{0, m}^{t}+\frac{1}{\gamma^{2}} \sum_{i=1}^{\gamma h}\left(\gamma h-i+\frac{1}{2}\right) b_{i, m}\right) t_{j, m}^{h} \\
\quad+\mu_{d}\left(v_{0, m}^{d}+h w_{0, m}^{d}+\frac{1}{\gamma^{2}} \sum_{i=1}^{\gamma h}\left(\gamma h-i+\frac{1}{2}\right) c_{i, m}\right) d_{j, m}^{h}, \\
\forall j \in \mathcal{J}_{m}, h \in \mathcal{H} \\
\epsilon_{j, m}^{h} \geq 0, \forall j \in \mathcal{J}_{m}, \forall h \in \mathcal{H}
\end{gathered}
$$

Clearly, $\epsilon_{j, m}^{h}=\left|e_{j, m}^{h}\right|$ in (6) - (9). Once the model parameters $\boldsymbol{v}_{\mathbf{0}}, \boldsymbol{w}_{\mathbf{0}}, \boldsymbol{a}$, $\boldsymbol{b}$, and $\boldsymbol{c}$ are obtained, a day-ahead hourly load forecast for a specified day in the same segment is computed by applying formula (10) to the corresponding 
temperature and dew point temperature forecast,

$$
\hat{l}_{j, m}^{h}=s_{m}(h)+\mu_{t} s_{t, m}(h) t_{j, m}^{h}+\mu_{d} s_{d, m}(h) d_{j, m}^{h}, \forall h \in \mathcal{H}, \forall j \in \mathcal{J}_{m}^{\prime}
$$

where $\mathcal{J}_{m}^{\prime}$ represents the set of days in segment $m \in \mathcal{M}$ in test set.

Parameters in the load model (5) can also be estimated by minimizing $\left\|e_{j, m}^{h}\right\|_{2}$ or $\left\|e_{j, m}^{h}\right\|_{\infty}$. The minimization of the fitted errors under $L_{2}$ norm results in a quadratic program but minimizing $\left\|e_{j, m}^{h}\right\|_{\infty}$, the maximum fitted error over the training set, leads to a linear program. The resulting linear program is modified by replacing $\epsilon_{j, m}^{h}$ with single decision variable $\epsilon$ in (7) - (9), and $\min _{\boldsymbol{v}_{\mathbf{0}}, \boldsymbol{w}_{\boldsymbol{0}}, \boldsymbol{a}, \boldsymbol{b}, \boldsymbol{c}} \epsilon$ in (6). This paper presents load forecast results from adopting the $L_{1}$ norm only, because it achieved greater accuracy in our tests.

An alternative approach to modeling the non-weather dependent load is to replace $s_{m}(h)$ by corresponding average load in hour $h$, as in [45]. However, TWE outperforms that method according to prediction accuracy and its stability.

\section{Data analysis and processing}

Our short-term load model, TWE, is applied to historical hourly load and corresponding weather forecast data in ISO-NE. Because the relationships between load and predictor variables; e.g., weather conditions, may gradually change over time, TWE is trained on a limited training period which ranged from April 2009 to March 2011, and validated on a test data set covering April 2011 to March 2012.

To improve the load prediction accuracy, seasons and segments of similar weather days in the same season are identified in Sections 3.1 and 3.2. Section 3.3 describes enrichment of load data to enhance the load prediction capability for the specified reference day. Section 3.4 discusses the other important factors considered in the load model.

\subsection{Division of seasons}

ISO-NE includes eight zones in six states. Among these zones, Connecticut (CT) accounts for about 25\%, the greatest share of electricity consumption in ISO-NE; North Central Massachusetts (NE) claims about 20\%, West Central Massachusetts (WC) 14\%, Southeast Massachusetts (SE) 12\%, Maine (ME) 9\%, New Hampshire (NH) 9\%, Rhode Island (RI) 7\%, and Vermont (VT) $4 \%$ of the load, respectively. Figure 1 presents hourly load and 
temperature variation ranges of these zones from April 2009 to March 2011. According to Fig. 1, zones CT and NE account for nearly half of the load in ISO-NE, and have a wider range than the other zones. The data analysis results are illustrated for $\mathrm{CT}$ because it has the largest and most variable loads. All eight zones in ISO-NE have similar temperature ranges.
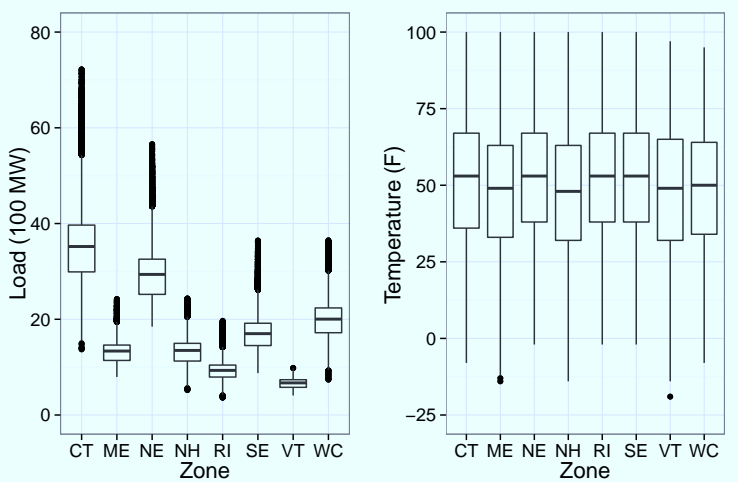

Figure 1: Hourly zonal load and temperature profiles in ISO-NE (Apr. 2009 - Mar. 2011)

To investigate which months can be grouped in the same season, scatter plots of electricity consumption level vs. temperature forecast are plotted in Fig. 2 for zone CT. The trend for each month is estimated by using simple linear regression of the actual load on the forecast temperature in the training set for each zone. The other zones have the similar trend observations to those in CT. In this paper, calendar months with similar linear trends of electricity consumption based on temperature are grouped together to form seasons. The temperature-based seasons of spring, summer, fall, and winter, are identified by applying $k$-means clustering on the slopes of the regression lines. Although electricity consumption may be more accurately captured by a nonlinear function of temperature in each month, the linear trends appear sufficient for clustering similar months. In Fig. 2, it appears that electricity consumption will quickly increase as temperature rises in summer (June through September), while decreasing with increasing temperature in winter (December through March).

\subsection{Days segmentation}

A short-term load model could be built for each season. However, Fig. 2 illustrates that the temperature varies in a wide range even in the same 

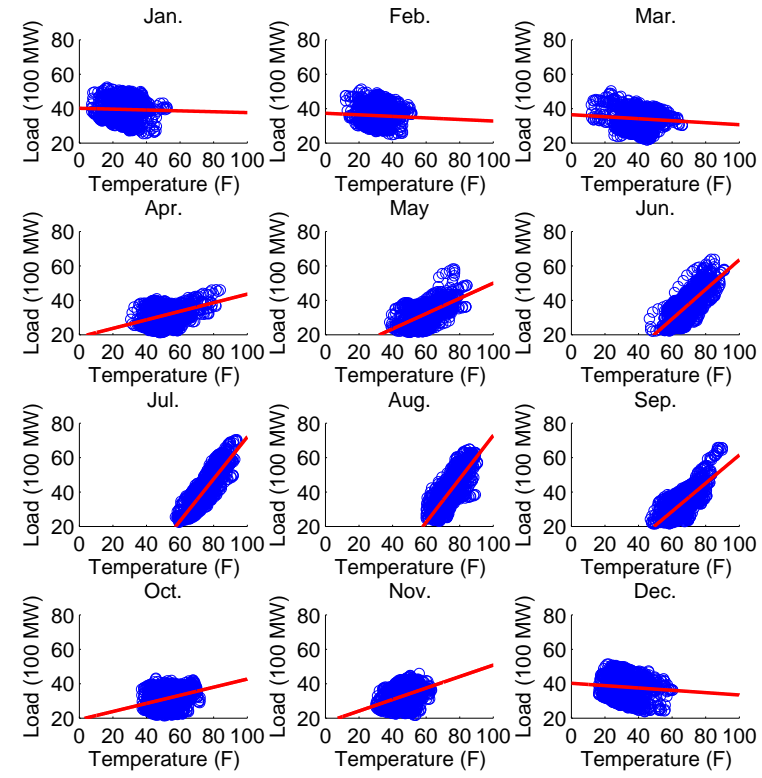

Figure 2: Scatter plot of load vs. temperature for each month in the training set, CT (Apr. 2009 - Mar. 2011) 
season. It is expected that the forecasting accuracy of a load model could be improved if days with similar temperature ranges in the same season were grouped together, as their daily load patterns could be similar. In this paper, all days in a season are divided into 3 segments: low, moderate, and high temperature days. A day is classified to one of the three segments according to the shortest $L_{2}$ distance between the day's average temperature and the three centroids identified by $k$-means clustering on the set of mean daily temperatures. Each of the resulting three segments covers all day types in a week. Because $k$-means clustering is an unsupervised learning method, it is appropriate to verify the clustering results by investigating the similarity in the same cluster as well as the differences among clusters. Fig. 3 displays the average daily load and temperature trajectories for each segment in summer. The widths of the corresponding $95 \%$ confidence intervals are smaller than $200 \mathrm{MWh}$ for the load trajectories, and smaller than $2 \mathrm{~F}$ for the temperature trajectories. The average daily temperature trajectory of each segment of days can be easily differentiated from the others. Moreover, the average daily load trajectories of the three segments differ significantly. Similar results are observed in the other zones for each season. Heating degree days (HDD) and cooling degree days (CDD) were also investigated as means to distinguish seasons and segment days. Because they resulted in the same seasons and similar segments to those discussed above, we did not include HDD/CDD in the short-term load model.

\subsection{Load data transformation}

Many short-term load modeling approaches $[4,32,36,43]$ identify an hourly load model for each day type but they require a large amount of historical data. A model that yields more reliable predictions may be built by using only recent data that better reflect current and near-future economic and demographic conditions; however, the quantity of such data is limited. This section presents a way to explore daily load pattern in a limited data set.

Average hourly load sequences for each day of the week in the moderate temperature segment are plotted for each season in Fig. 4, which shows that the load pattern in each day type in the same season tends to be similar, although the load levels in weekend days are lower than those in weekdays. Based on this similarity in load shapes, we enrich the data in each segment by applying a transformation among day types for each season. Here we detail the data transformation procedure briefly mentioned in [45]. 

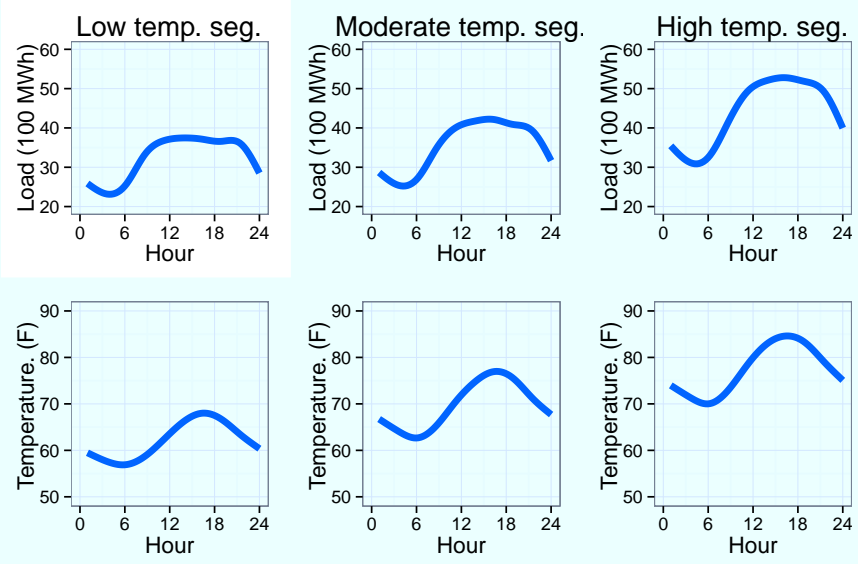

Figure 3: Average daily load and temperature trajectories for three segments in summer, CT (Apr. 2009 - Mar. 2011)

Let $\mathcal{D}$ be the set of days of a specified day type in a segment within a season, and $\mathcal{D}^{\prime}$ be the set of days of another day type in the same segment of the same season. Suppose $t_{\omega} \in \mathbb{R}^{24}$ is a sequence of hourly temperature forecast values in a day $\omega \in \mathcal{D}$, and let $\psi_{o}: \mathbb{R}^{24} \rightarrow \mathbb{R}^{24}$ be an operator that maps $t_{\omega}$ to the corresponding sequence of hourly load $l_{\omega} \in \mathbb{R}^{24}$ :

$$
l_{\omega}=\psi_{o}\left(t_{\omega}\right)+e_{\omega}, \forall \omega \in \mathcal{D}
$$

so that $\left\|e_{\omega}\right\|$ is minimized over $\mathcal{D}$. Then

$$
\tilde{l}_{\omega^{\prime}}=\psi_{o}\left(t_{\omega^{\prime}}\right), \forall \omega^{\prime} \in \mathcal{D}^{\prime}
$$

simulates a possible sequence of hourly loads $\tilde{l}_{\omega^{\prime}}$, if the same sequence of temperature forecasts of $\omega^{\prime} \in \mathcal{D}^{\prime}$ were applied to $\mathcal{D}$. Once the simulated possible sequences of hourly load for each day in day type set $\mathcal{D}^{\prime}$ are obtained, a transformation between $\left\{\tilde{l}_{\omega^{\prime}}\right\}$ and $\left\{l_{\omega^{\prime}}\right\}$ can be found.

There are many possible approaches to transforming $l_{\omega^{\prime}}$ to $\tilde{l}_{\omega^{\prime}}$, but for easily transforming $\tilde{l}_{\omega^{\prime}}$ back to $l_{\omega^{\prime}}$ for the purpose of prediction in the original scale, the transform function is restricted to be linear:

$$
\tilde{l}_{\omega^{\prime}}=\Lambda l_{\omega^{\prime}}+\zeta_{\omega^{\prime}}, \forall \omega^{\prime} \in \mathcal{D}^{\prime}
$$


where $\Lambda$ is a matrix obtained by minimizing the error vectors $\left\{\zeta_{\omega^{\prime}}\right\}$ under a specified norm; i.e., $\|\cdot\|_{L_{1}}$. In addition, the transformation matrix is further restricted to be diagonal to avoid singularity when computing $\Lambda^{-1}$. Although any day in a week can be viewed as a reference day type, we use Wednesday as the reference day-type to minimize the influences of weekends.
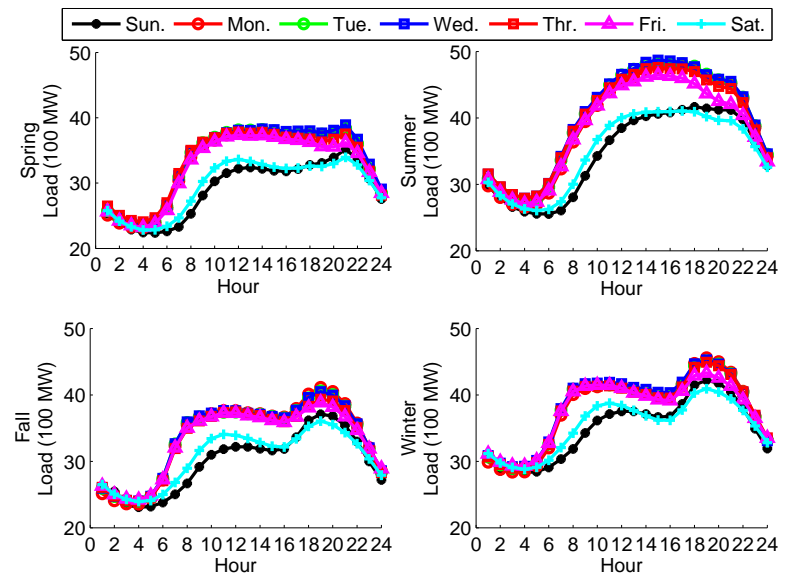

Figure 4: Average daily load sequence in each day type in moderate temperature segment in Apr. 2009 - Mar. 2011, CT

By enriching data for a specified data type, more diverse weather forecast records and their corresponding loads enhance the model prediction reliability for the specified day type. Once load forecasts for the specified day-type are generated, load forecasts for the other day types can be generated according to the linear transformation $\Lambda^{-1}$. The linear transformations on load values between the specified day type and the other day types have certain transformation errors, which are accounted for in the prediction errors.

\subsection{Important load forecasting factors}

In addition to temperature, some other factors can also influence the electricity demand level. In Fig. 5, scatter plots of hourly load and the corresponding humidity forecast, measured by dew point temperature, are plotted for different temperature ranges in summer. The electricity consumption increases with the forecast dew point temperature across days with similar temperature forecasts. Therefore, dew point temperature is included as another factor in the short-term load model in summer. Such a relationship 
does not appear in other seasons, but other factors, such as wind speed and cloud cover, might be included if data are available.
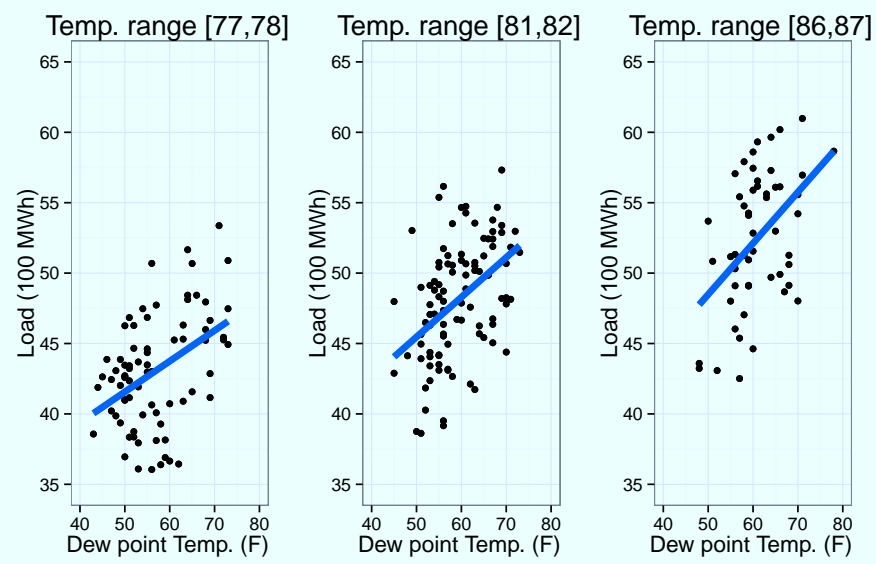

Figure 5: Load vs. dew point temperature (humidity) forecast for hours $15 \sim 16$ within specified range of temperature forecast in summer, CT

While weather is an important contributor to electricity consumption, the STLF model must also account for temporal patterns. Fig. 6 illustrates how the temporal patterns of both temperature and load vary by season. These variations confirm the need for the additional epi-spline, $s_{m}(h)$, to approximate the temporal dependence separately in each segment of each season.

\section{Case study}

Hourly load data and the corresponding weather forecasts were collected from each zone in ISO-NE for the time period ranging from April 2009 to March 2012. As the previous papers have discussed, public holidays must be differentiated from regular weekdays because they have different electricity consumption patterns. In this paper, New Year's Day, Memorial Day, Independence Day, Labor Day, Thanksgiving, and Christmas are all considered as Sunday [4]. Moreover, because of power system outages that resulted from the extreme weather conditions, days ranging from 2010-2-5 to 2010-27, 2011-8-28 to 2011-8-31, and 2011-10-27 to 2011-11-4 were not considered in the short-term load model. Experimentation revealed that a slight shift in 

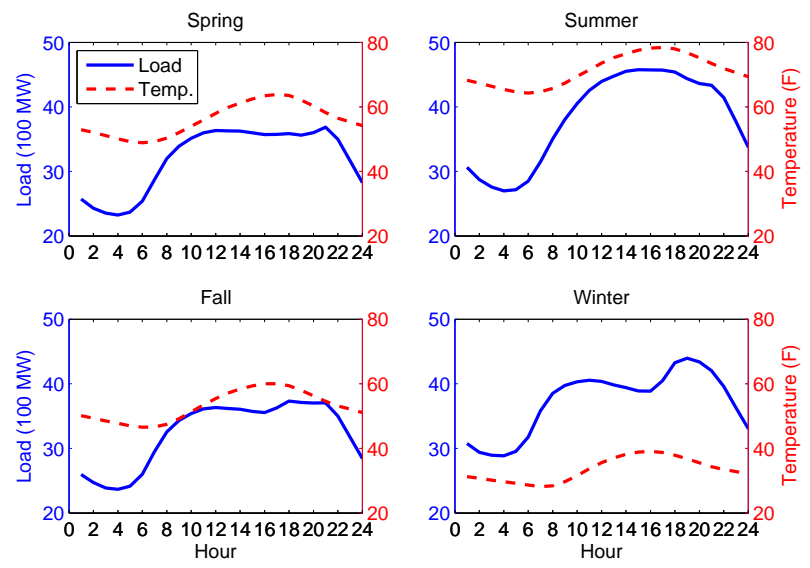

Figure 6: Average daily load and average daily temperature trajectories in Apr. 2009 Mar. 2011, CT

\begin{aligned} & \multicolumn{2}{c}{ Table 1: Refined seasons } \\ & \hline \hline Season name Range \\ & \hline Spring (Spr.) Apr.1 - May.14 \\ & Summer (Sum.) May.15 - Sep.14 \\ & Fall (Fal.) Sep.15 - Nov.30 \\ & Winter (Win.) Dec.1 - Mar.31 \\ & \hline\end{aligned}

the division points between the four seasons, as described in Table. 1, would improve the prediction accuracy of TWE.

In the model training process, weights $\mu_{t}$ and $\mu_{d}$ were set to 0.5 for summer; otherwise, $\mu_{d}$ was set to 0 because dew point temperature does not drive load in the other seasons. In the validation procedure, the trained model TWE was applied to obtain 24-hour load predictions for all 8 zones in ISO-NE all at once for each day, given the day-ahead 24-hour weather forecast. Model TWE was implemented in Python and Pyomo [49] on a Dell laptop with 6GB RAM.

In practice, polynomials are frequently used to depict the nonlinear relationships between load and weather conditions [50, 51, 52]. Two recent representative regression based short-term load models [4, 42] are considered as benchmarks to show the competitive performance of TWE in capturing the nonlinear relationship between temperature forecast and load, while attaining a narrower band of prediction errors. Both benchmark approaches 
identify a load model for each hour in a day type for each month or each season. The two load models are given in (14) and (15), and called the Hong and Charlton \& Singleton (C\&S) models, respectively. The Hong model is given by:

$$
\begin{aligned}
l_{j}^{h}= & \beta_{0}+\beta_{1} T+\beta_{2} D_{i(j)} H_{h}+\beta_{3} M_{k(j)}+\beta_{4} M_{k(j)}\left(t_{j}^{h}\right)+\beta_{5} M_{k(j)}\left(t_{j}^{h}\right)^{2} \\
& +\beta_{6} M_{k(j)}\left(t_{j}^{h}\right)^{3}+\beta_{7} H_{h}\left(t_{j}^{h}\right)+\beta_{8} H_{h}\left(t_{j}^{h}\right)^{2}+\beta_{9} H_{h}\left(t_{j}^{h}\right)^{3}+e_{j}^{h}, \\
& \forall j \in \mathcal{J}_{H}, h \in \mathcal{H}
\end{aligned}
$$

where $T$ is the load increasing trend, and $\mathcal{J}_{H}$ is the days set in training set. $D_{i(j)}, i(j)=1, \cdots 7, H_{h}, h=1, \cdots, 24$ and $M_{k(j)}, k(j)=1, \cdots, 12$ are dummy variables representing 7 day types in a week, 24 hours in a day, and 12 months in a year, respectively.

The C\&S model divides a year into four seasons: spring, summer, fall and winter; and divides days into two types: weekday and weekend. For each hour of a day type in a season, it is given as follows:

$$
\begin{aligned}
l_{j}^{h}= & \alpha_{1}+\alpha_{2} \tau_{j}+\alpha_{3}\left(t_{j}^{h}\right)+\alpha_{4}\left(t_{j}^{h}\right) \tau_{j}+\alpha_{5}\left(t_{j}^{h}\right)^{2}+\alpha_{6}\left(t_{j}^{h}\right)^{2} \tau_{j}+\alpha_{7} \tau_{j}^{\prime}+\alpha_{8}\left(t_{j}^{h}\right) \tau_{j}^{\prime} \\
& +\alpha_{9}\left(t_{j}^{h}\right)^{2} \tau_{j}^{\prime}+e_{j}^{h}, \forall j \in \mathcal{J}_{C S}, h \in \mathcal{H}
\end{aligned}
$$

where $\mathcal{J}_{C S}$ denotes the days set of a given day type in a specified season, $\tau_{j}$ the day number in the sequence of days, and $\tau_{j}^{\prime}$ the day number within the specified season. The above two benchmark models, (14) and (15), were both implemented in R. Charlton and Singleton employed several other approaches to improve performance of the short-term load model, including using forecasts from multiple weather stations instead of one, applying a local average technique, and smoothing the temperature forecast [42]. To compare models on the same basis, we did not incorporate these techniques to forecast ISO-NE load in the test set. However, these useful techniques could be applied to improve the accuracy of hourly load forecasting in future work.

Because of the flexibility of epi-splines to model high nonlinearity, we expect that TWE could provide higher quality hourly load prediction than ordinary polynomial regression models do. To test this hypothesis, we measure prediction quality in terms of prediction accuracy, stability of prediction 
accuracy, and skewness of prediction error distributions. Similarly to [53, 42], weighted error measurements are applied to investigate the quality of shortterm load prediction in this paper. Weighted mean absolute percentage error (WMAPE) is used to emphasize the prediction accuracy in the hours in which most electricity is consumed. Weights $\left\{\rho_{h}\right\}, h \in \mathcal{H}$, are calculated as:

$$
\rho_{h}=\frac{\sum_{j \in \mathcal{J}^{\prime}} l_{j}^{h}}{\sum_{j \in \mathcal{J}^{\prime}} \sum_{h \in \mathcal{H}} l_{j}^{h}}, \forall h \in \mathcal{H}
$$

where $\mathcal{J}^{\prime}$ denotes the set of test days. Following (16), WMAPE is defined as:

$$
\mathrm{WMAPE}=\frac{\sum_{j \in \mathcal{J}^{\prime}} \sum_{h \in \mathcal{H}} \rho_{h}\left|e_{j}^{h}\right|}{\sum_{j \in \mathcal{J}^{\prime}} \sum_{h \in \mathcal{H}} \rho_{h}} .
$$

Weighted root mean square error (WRMSE) is applied to investigate the stability of prediction accuracy. The WRMSE is defined similarly as in [53]:

$$
\text { WRMSE }=\sqrt{\frac{\sum_{j \in \mathcal{J}^{\prime}} \sum_{h \in \mathcal{H}} \rho_{h}\left(e_{j}^{h}\right)^{2}}{\sum_{j \in \mathcal{J}^{\prime}} \sum_{h \in \mathcal{H}} \rho_{h}}} .
$$

Densities of prediction errors are displayed to check for skewness.

Figure 7 displays WMAPEs for each zone in ISO-NE for all seasons in the test set. In spring, summer, and fall, TWE provides the most accurate predictions in nearly all of the zones. Compared to Hong and C\&S models, TWE better captures load consumption seasonality and the complex nonlinear relationship between load and weather in these seasons. In winter, hourly load prediction from TWE is slightly less accurate than the better one of the other two methods. The lower accuracy of TWE in winter results from its lower prediction accuracy in the high temperature segment. In contrast, both of the benchmark approaches, build a model for each hour in each month/season. C\&S model even takes into account the order a day takes in a season. This strategy results in more accurate hourly load prediction in high temperature segment of winter days. Although TWE does not predict as accurately as the winner between Hong and C\&S models in winter, TWE demonstrates the most accurate prediction overall.

WRMSEs for each zone are presented in Fig. 8. TWE results in smaller WRMSE in most of the zones, especially in the highest consumption zones, CT and NE. In zone NH, the WRMSE from TWE is higher than that of the 

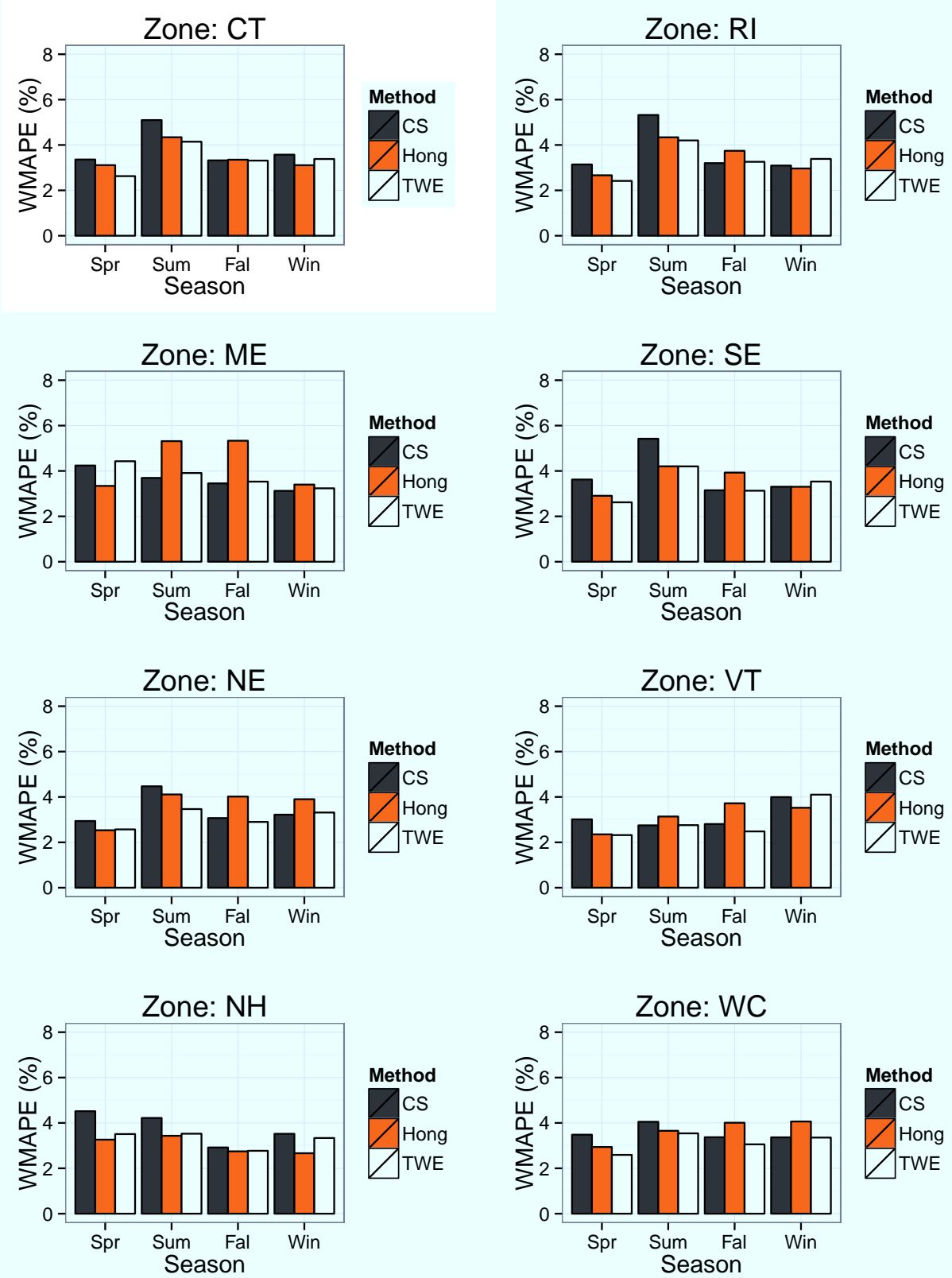

Figure 7: WMAPEs of TWE and other two benchmark models in Apr. 2011 - Mar. 2012 
Hong model, which is mainly caused by the less accurate prediction of TWE in the high temperature segment in winter. The prediction errors of TWE in hours 10 to 21 in a warm winter day are smaller than those from the Hong model, but they are larger at night. Outside zone NH, zonal load prediction accuracy of TWE is more stable than the other two benchmark models as measured by WRMSE.

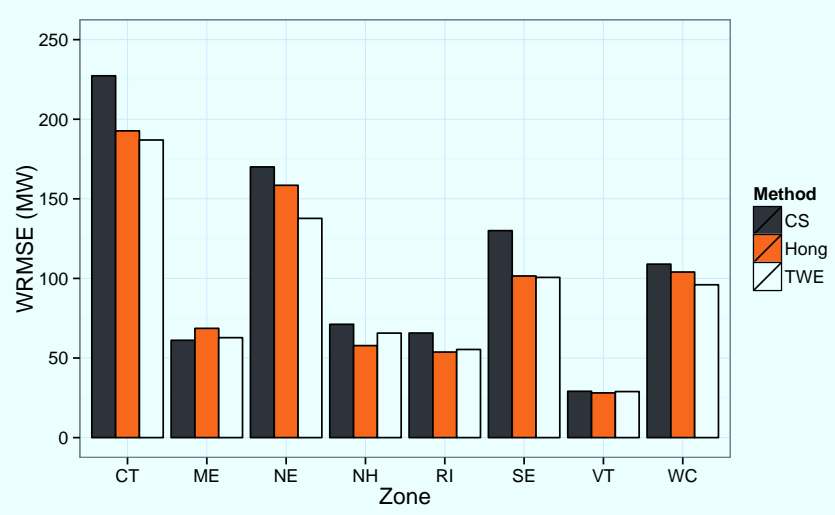

Figure 8: WRMSEs of TWE and other two benchmark models in Apr. 2011 - Mar. 2012

Prediction error distributions, in the form of densities fitted by kernel density estimation in $\mathrm{R}$, are plotted at three-hour intervals in Fig. 9. To save space they are presented for CT only, but similar results were observed in the other load zones. Compared to TWE, the benchmark models produce flatter prediction error densities with longer and thicker tails, especially in the daytime hours 10 to 21, during which most residential, commercial, and industrial electricity demands occur. In addition, the densities of errors from Hong and C\&S are more skewed to either the left or the right than those from TWE during the daytime. At night, all of the three models share very similar densities of prediction errors.

According to the above comparisons at zonal level, TWE generally has the best prediction accuracy in all zones, all seasons, except for winter. In contrast to Hong and C\&S models, TWE also provides more stable prediction accuracy in high-load zones, and similarly stable prediction accuracy in the other zones. TWE provides less biased predictions in most zones and hours.

The performance of TWE, Hong and C\&S models are also compared at the regional level. Fig. 10 summarizes the distributions of prediction errors 
Hour: 3

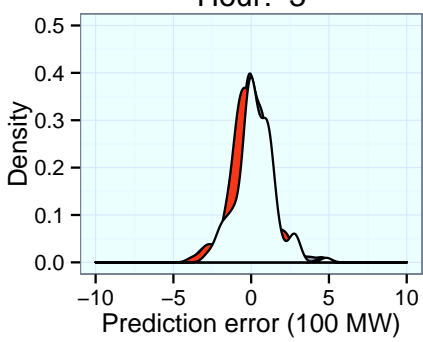

Hour: 6

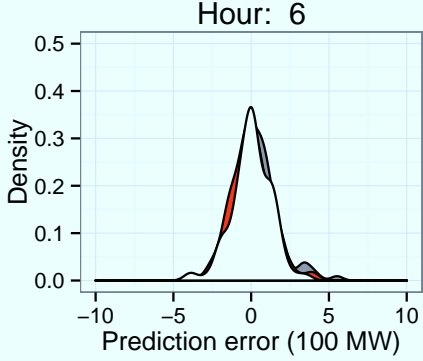

Hour: 9

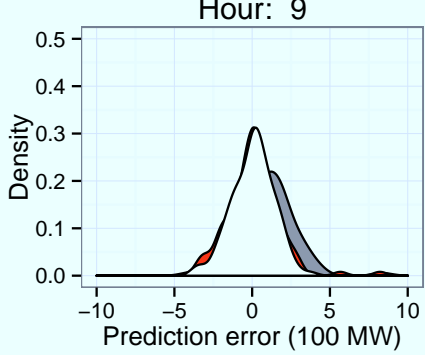

Hour: 12

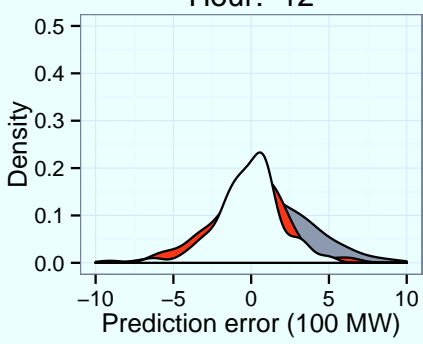

Hour: 15

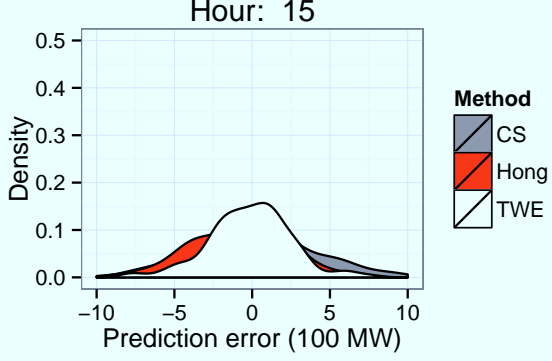

Hour: 18

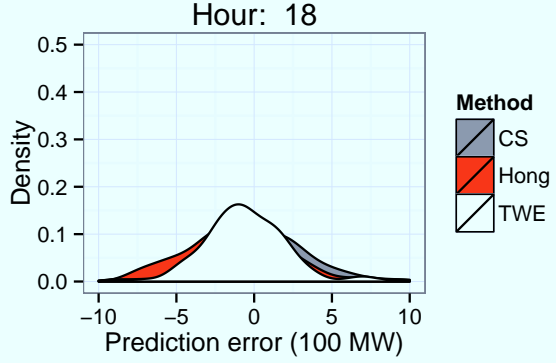

Hour: 21

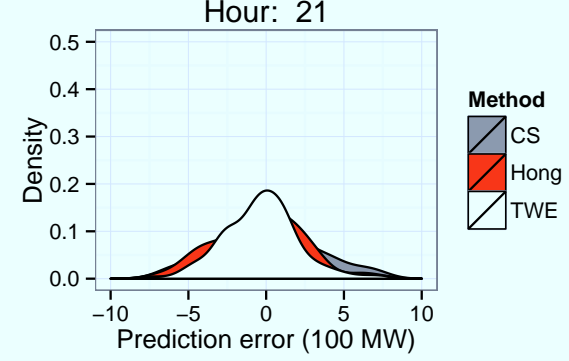

Hour: 24

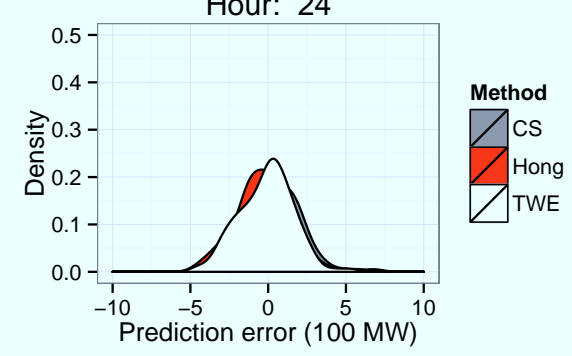

Figure 9: Densities of prediction errors in summer in 2011 - 2012 for TWE and the benchmark models: zone CT 
in the test set for each model over ISO-NE. TWE yields narrower and less skewed distributions of prediction errors, compared to the benchmark models.

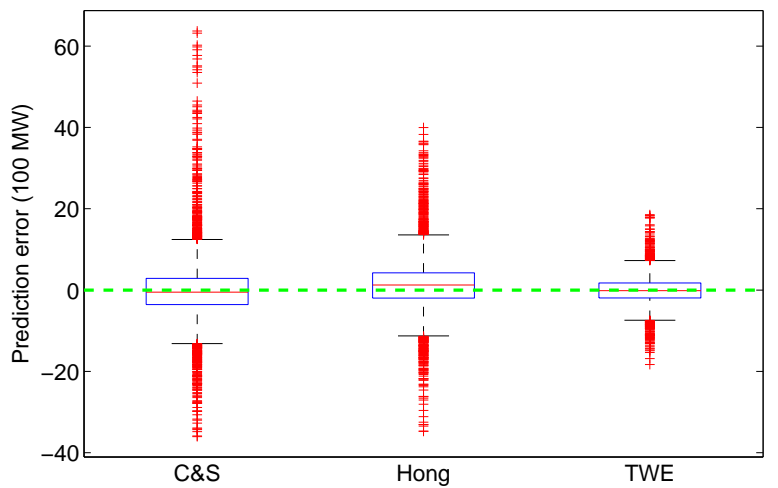

Figure 10: Box plot of prediction errors of TWE and two benchmark models for ISO-NE in test set (Apr. 2011 - Mar. 2012)

The autocorrelation function (ACF) is commonly used to compute serial correlations of a time series, but may be distorted in this application when lags span parts of consecutive days to which different weather forecasts apply. To restrict the attention to only the serial correlations among load values in the same day, a modified ACF, called intraday ACF (InACF), is applied to measure the serial correlations. The sample InACF at lag $\kappa$ for load forecast $\left\{\hat{l}_{j}^{h}\right\}_{\mathcal{J}^{\prime}}^{\mathcal{H}}$ from a specified load model is calculated as:

$$
\hat{\lambda}_{\kappa}=\frac{\sum_{j \in \mathcal{J}^{\prime}} \sum_{h=1}^{24-\kappa}\left(\hat{l}_{j}^{h}-\hat{\bar{l}}\right)\left(\hat{l}_{j}^{h+\kappa}-\hat{\bar{l}}\right)}{\sum_{j \in \mathcal{J}^{\prime}} \sum_{h=1}^{24}\left(\hat{l}_{j}^{h}-\hat{\bar{l}}\right)^{2}}, \kappa=1,2, \cdots, 23
$$

where $\hat{\bar{l}}$ is the average value of series $\left\{\hat{l}_{j}^{h}\right\}$.

The sample InACF, $\hat{\lambda}_{\kappa}^{\prime}, \kappa=1,2, \cdots, 23$, is also calculated for actual hourly load in the test set for comparison, as displayed in Fig. 11. The InACF in Fig. 11 indicates that daily load trajectories from TWE and the two benchmark models have very similar serial correlations to those from the actual load in a day. The mean absolute errors are plotted by hour in 
Fig. 12 to check whether the forecast daily load trajectories are realistic. From Fig. 12, TWE predictions are closer to the actual trajectory in spring, summer, and fall, in hours 10 to 21 . In winter, TWE produces similar daily forecast load trajectory to the one from C\&S. The daily load trajectory from TWE is better than that from the Hong model in most of the hours between 10 and 21, but worse in hours 17 to 19.

Because TWE provides more accurate predictions than the benchmark models, as well as more stable prediction accuracy and less skewed prediction intervals overall both at the zonal level and the ISO-NE level, it is expected that the total cost of unit commitment and economic dispatch that result from the day-ahead hourly load trajectory could satisfy the economic requirement of utilities and independent system operators. In addition, probabilistic scenarios for the next day's 24-hour load sequence would be more realistic, because TWE also emphasizes the temporal pattern.

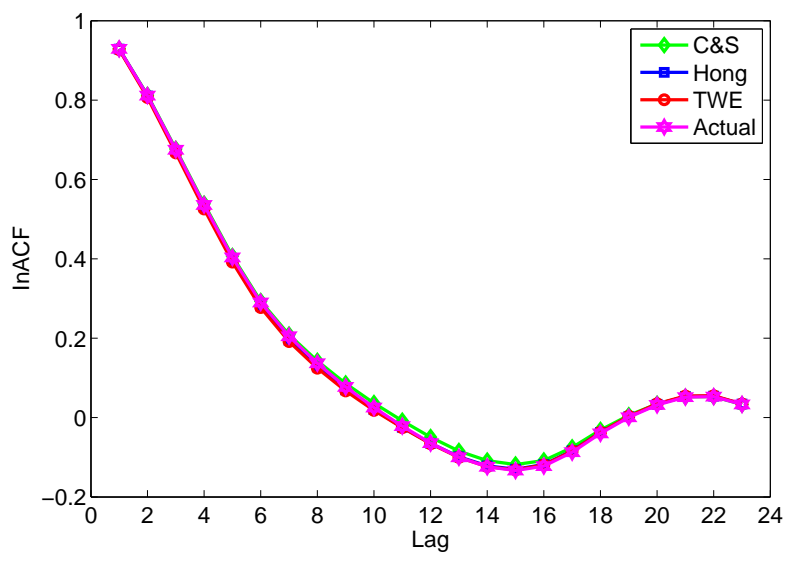

Figure 11: InACF of actual and forecast daily load trajectory of ISO-NE in test set (Apr. 2011 - Mar. 2012)

\section{Conclusion}

In this paper, a short-term load model, TWE, is developed to use weather forecasts as input and capture temporal patterns using functional approximation. Two recent representative regression short-term load models are selected to compare distributions of prediction errors. Compared to the 

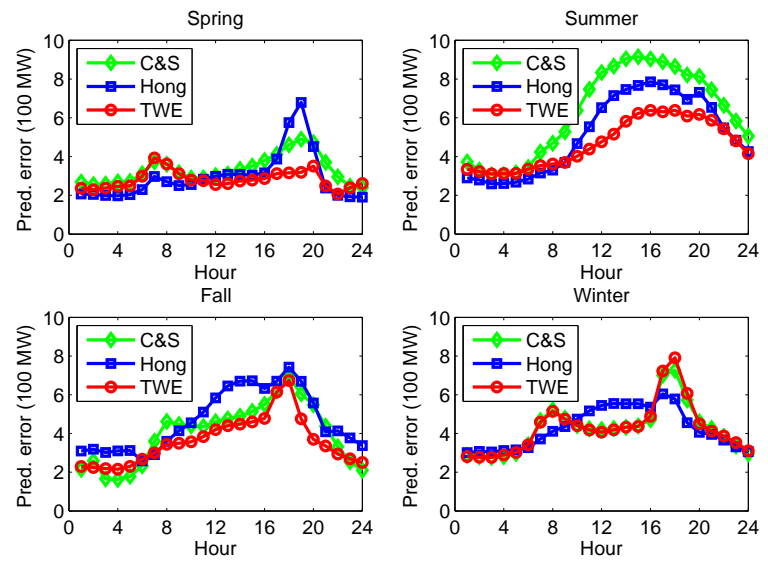

Figure 12: Mean absolute errors in daily load trajectory of ISO-NE in test set (Apr. 2011 - Mar. 2012)

benchmarks, TWE not only obtains less biased and narrower distributions of prediction errors, but also preserves similar serial correlations among forecasted hourly load values to the actual ones. From the perspective of accuracy in each hour and relationships among hourly loads, TWE is expected to be appropriate for both providing regional day-ahead hourly load prediction for power system generation, and generating practical regional load scenarios for day-ahead stochastic unit commitment given temperature and dew point temperature forecasts. Comparing the commitment cost and expected generation cost that result from the generated load scenarios to the cost based on the actual load is an important criterion for measuring the quality of scenarios, which will be addressed in future work.

The quality of load prediction by TWE in winter can be further improved from two aspects: including more external factors and improving the model. Prediction accuracy might be improved by considering the chilling effect from wind speed in winter and mitigating effect of sunshine in the TWE model. The additive structure of TWE allows more factors to be considered easily. In addition, the gradient boosting framework $[54,55]$ could be combined with TWE, so that the weather dependent load component would be better fitted with a sequence of weak weather predictors.

TWE can also be applied to power systems with deep penetration of behind-the-meter distributed generation, but several other factors must be 
included in the model when solar and wind energy are the major sources of distributed generation. These additional external factors include sunshine hours, cloud cover, rainfall, snow, and wind speed. On the other hand, modeling load in the presence of price-based demand response would require a more sophisticated approach to capture the demand-price relationship.

\section{Acknowledgement}

Funding for this work was provided by the U.S. Department of Energy ARPA-e program under the Green Energy Network Integration (GENI) program.

We thank Yihua Li for her help with developing $\mathrm{R}$ codes.

\section{References}

[1] F. Andersen, H. V. Larsen, R. Gaardestrup, Long term forecasting of hourly electricity consumption in local areas in Denmark, Applied Energy 110 (2013) 147-162.

[2] M. De Felice, A. Alessandri, F. Catalano, Seasonal climate forecasts for medium-term electricity demand forecasting, Applied Energy 137 (2015) 435-444.

[3] J. W. Taylor, P. E. McSharry, Short-term load forecasting methods: An evaluation based on European data, IEEE Transactions on Power Systems 22 (4) (2007) 2213-2219.

[4] T. Hong, Short term electric load forecasting, Ph.D. thesis, North Carolina State University (2011).

[5] A. Goia, C. May, G. Fusai, Functional clustering and linear regression for peak load forecasting, International Journal of Forecasting 26 (4) (2010) 700-711.

[6] G. Sudheer, A. Suseelatha, Short term load forecasting using wavelet transform combined with holt-winters and weighted nearest neighbor models, International Journal of Electrical Power \& Energy Systems 64 (2015) 340-346. 
[7] S. Takriti, J. R. Birge, E. Long, A stochastic model for the unit commitment problem, IEEE Transactions on Power Systems 11 (3) (1996) $1497-1508$.

[8] L. Wu, M. Shahidehpour, T. Li, Stochastic security-constrained unit commitment, IEEE Transactions on Power Systems 22 (2) (2007) 800811.

[9] P. A. Ruiz, C. R. Philbrick, E. Zak, K. W. Cheung, P. W. Sauer, Uncertainty management in the unit commitment problem, IEEE Transactions on Power Systems 24 (2) (2009) 642-651.

[10] K. Cheung, D. Gade, C. S. Monroy, S. M. Ryan, J.-P. Watson, R. J.B. Wets, et al., Toward scalable stochastic unit commitment - Part 2: Assessing solver performance, Energy Systems 6 (3) (2015) 417-438.

[11] D. Bertsimas, E. Litvinov, X. A. Sun, J. Zhao, T. Zheng, Adaptive robust optimization for the security constrained unit commitment problem, IEEE Transactions on Power Systems 28 (1) (2013) 52-63.

[12] R. Jiang, J. Wang, Y. Guan, Robust unit commitment with wind power and pumped storage hydro, IEEE Transactions on Power Systems 27 (2) (2012) 800-810.

[13] Y. Guan, J. Wang, Uncertainty sets for robust unit commitment, IEEE Transactions on Power Systems 29 (3) (2014) 1439-1440.

[14] Y. Liu, M. C. Ferris, F. Zhao, T. Zheng, E. Litvinov, A stochastic unit commitment with derand technique for ISO's reserve adequacy assessment, in: 2015 IEEE Power \& Energy Society General Meeting, IEEE, 2015, pp. 1-5.

[15] S. Fan, L. Chen, W.-J. Lee, Short-term load forecasting using comprehensive combination based on multimeteorological information, IEEE Transactions on Industry Applications 45 (4) (2009) 1460-1466.

[16] Y. Chen, P. B. Luh, C. Guan, Y. Zhao, L. D. Michel, M. A. Coolbeth, et al., Short-term load forecasting: Similar day-based wavelet neural networks, IEEE Transactions on Power Systems 25 (1) (2010) 322-330. 
[17] M. Mohandes, Support vector machines for short-term electrical load forecasting, International Journal of Energy Research 26 (4) (2002) 335345 .

[18] Y. Wang, Q. Xia, C. Kang, Secondary forecasting based on deviation analysis for short-term load forecasting, IEEE Transactions on Power Systems 26 (2) (2011) 500-507.

[19] J. Che, J. Wang, Short-term load forecasting using a kernel-based support vector regression combination model, Applied Energy 132 (2014) 602-609.

[20] E. A. Feinberg, D. Genethliou, Load forecasting, in: Applied Mathematics for Restructured Electric Power Systems, Springer, 2005, pp. 269-285.

[21] E. Kyriakides, M. Polycarpou, Short term electric load forecasting: A tutorial, in: Trends in Neural Computation, Springer, 2007, pp. 391418.

[22] S. Fan, L. Chen, Short-term load forecasting based on an adaptive hybrid method, IEEE Transactions on Power Systems 21 (1) (2006) 392-401.

[23] H. Nie, G. Liu, X. Liu, Y. Wang, Hybrid of ARIMA and SVMs for short-term load forecasting, Energy Procedia 16 (2012) 1455-1460.

[24] R.-A. Hooshmand, H. Amooshahi, M. Parastegari, A hybrid intelligent algorithm based short-term load forecasting approach, International Journal of Electrical Power \& Energy Systems 45 (1) (2013) 313-324.

[25] N. Liu, Q. Tang, J. Zhang, W. Fan, J. Liu, A hybrid forecasting model with parameter optimization for short-term load forecasting of microgrids, Applied Energy 129 (2014) 336-345.

[26] W. P. Mahoney, K. Parks, G. Wiener, Y. Liu, W. L. Myers, J. Sun, et al., A wind power forecasting system to optimize grid integration, IEEE Transactions on Sustainable Energy 3 (4) (2012) 670-682.

[27] ISO New England, Create demand forecast, http://www.isone.com/rules_proceds/operating/sysop/out_sched/sop_outsch_0040_0010.pdf (2013). 
[28] R. K. Jain, K. M. Smith, P. J. Culligan, J. E. Taylor, Forecasting energy consumption of multi-family residential buildings using support vector regression: Investigating the impact of temporal and spatial monitoring granularity on performance accuracy, Applied Energy 123 (2014) 168178.

[29] C. R. Touretzky, R. Patil, Building-level power demand forecasting framework using building specific inputs: Development and applications, Applied Energy 147 (2015) 466-477.

[30] A. Vaghefi, M. Jafari, E. Bisse, Y. Lu, J. Brouwer, Modeling and forecasting of cooling and electricity load demand, Applied Energy 136 (2014) 186-196.

[31] N. D. Uri, Forecasting peak system load using a combined time series and econometric model, Applied Energy 4 (3) (1978) 219-227.

[32] N. Amjady, Short-term hourly load forecasting using time-series modeling with peak load estimation capability, IEEE Transactions on Power Systems 16 (3) (2001) 498-505.

[33] S.-J. Huang, K.-R. Shih, Short-term load forecasting via ARMA model identification including non-gaussian process considerations, IEEE Transactions on Power Systems 18 (2) (2003) 673-679.

[34] J. W. Taylor, Short-term electricity demand forecasting using double seasonal exponential smoothing, Journal of the Operational Research Society 54 (8) (2003) 799-805.

[35] J. W. Taylor, Triple seasonal methods for short-term electricity demand forecasting, European Journal of Operational Research 204 (1) (2010) $139-152$.

[36] J. M. Liu, R. Chen, L.-M. Liu, J. L. Harris, A semi-parametric time series approach in modeling hourly electricity loads, Journal of Forecasting 25 (8) (2006) 537-559.

[37] A. D. Papalexopoulos, T. C. Hesterberg, A regression-based approach to short-term system load forecasting, IEEE Transactions on Power Systems 5 (4) (1990) 1535-1547. 
[38] R. F. Engle, C. Mustafa, J. Rice, Modelling peak electricity demand, Journal of Forecasting 11 (3) (1992) 241-251.

[39] T. Haida, S. Muto, Regression based peak load forecasting using a transformation technique, IEEE Transactions on Power Systems 9 (4) (1994) 1788-1794.

[40] W. Charytoniuk, M.-S. Chen, P. Van Olinda, Nonparametric regression based short-term load forecasting, IEEE Transactions on Power Systems 13 (3) (1998) 725-730.

[41] J. D. Black, Load hindcasting: A retrospective regional load prediction method using reanalysis weather data, Master's thesis, University of Massachusetts Amherst (2011).

[42] N. Charlton, C. Singleton, A refined parametric model for short term load forecasting, International Journal of Forecasting 30 (2) (2014) 364368.

[43] S. Fan, R. J. Hyndman, Short-term load forecasting based on a semiparametric additive model, IEEE Transactions on Power Systems 27 (1) (2012) 134-141.

[44] Y. Feng, D. Gade, S. M. Ryan, J.-P. Watson, R. J. Wets, D. L. Woodruff, A new approximation method for generating day-ahead load scenarios, in: 2013 IEEE Power and Energy Society General Meeting, IEEE, 2013, pp. 1-5.

[45] Y. Feng, I. Rios, S. M. Ryan, K. Spurkel, J.-P. Watson, R. J.-B. Wets, et al., Toward scalable stochastic unit commitment - Part 1: Load scenario generation, Energy Systems 6 (3) (2015) 309-329.

[46] I. Rios, R. J.-B. Wets, D. L. Woodruff, Multi-period forecasting and scenarios generation with limited data, Computational Management Science 12 (2) (2015) 267-295.

[47] R. J.-B. Wets, S. W. Bianchi, Term and volatility structures, in: Handbook of Asset and Liability Management: Theory and Methodology, Elsevier, 2006, pp. 26-28. 
[48] J. O. Royset, R. J.-B. Wets, From data to assessments and decisions: Epi-spline technology, in: Tutorials in Operations Research: Bridging Data and Decision, INFORMS, 2014, pp. 27-53.

[49] Sandia National Laboratories, Pyomo, https://software.sandia.gov/trac/coopr/wiki/Pyomo (2014).

[50] M. T. Hagan, S. M. Behr, The time series approach to short term load forecasting, IEEE Transactions on Power Systems 2 (3) (1987) 785-791.

[51] J. Moral-Carcedo, J. Vicens-Otero, Modelling the non-linear response of spanish electricity demand to temperature variations, Energy Economics 27 (3) (2005) 477-494.

[52] T. Hong, P. Wang, H. L. Willis, A naïve multiple linear regression benchmark for short term load forecasting, in: 2011 IEEE Power and Energy Society General Meeting, IEEE, 2011, pp. 1-6.

[53] T. Hong, P. Pinson, S. Fan, Global energy forecasting competition 2012, International Journal of Forecasting 30 (2) (2014) 357-363.

[54] P. Bühlmann, B. Yu, Boosting with the $L_{2}$ loss: Regression and classification, Journal of the American Statistical Association 98 (462) (2003) 324-339.

[55] P. Bühlmann, T. Hothorn, Boosting algorithms: Regularization, prediction and model fitting, Statistical Science (2007) 477-505. 